\title{
Anabases
}

ANABASES Traditions et réceptions de l'Antiquité

$15 \mid 2012$

Varia

\section{Les « musées de papier » ou le règne quasi sans partage de l'in-folio illustré}

\section{Magali Soulatges}

\section{(2) OpenEdition}

\section{Journals}

Édition électronique

URL : http://journals.openedition.org/anabases/3739

DOI : 10.4000/anabases.3739

ISSN : 2256-9421

Éditeur

E.R.A.S.M.E.

Édition imprimée

Date de publication : 1 avril 2012

Pagination : 129-142

ISSN : 1774-4296

\section{Référence électronique}

Magali Soulatges, «Les « musées de papier » ou le règne quasi sans partage de l'in-folio illustré »,

Anabases [En ligne], 15 | 2012, mis en ligne le 01 avril 2015, consulté le 21 octobre 2019. URL : http:// journals.openedition.org/anabases/3739; DOI : 10.4000/anabases.3739

(c) Anabases 


\title{
Les " musées de papier" ou le règne quasi sans partage de l'in-folio illustré
}

\author{
Magali Soulatges
}

"Son goût pour l'Antiquité, et puisqu'il faut le dire, l'état de sa fortune et la nécessité qui font tant de métamorphoses en ce monde, ont entraîné [l'auteur] dans cette entreprise, qui a été bien plus loin qu'il n'avait imaginé ; ainsi, on lui pardonnera d'être auteur de quatre in-folio, puisque c'est malgré lui qu'il l'est devenu. " D’Hancarville, Antiquités étrusques, grecques et romaines, Préface

UNE « SAISON XVIII $^{\mathrm{e}}$ " s'est tenue au Louvre de septembre 2010 à avril 2011, déclinée en quatre expositions à visées complémentaires ${ }^{1}$. Parmi elles, l'exposition "Musées de papier : l'Antiquité en livres (1600-1800) », conçue par Élisabeth Décultot, a confronté le visiteur à une approche inhabituelle des antiquités, avec un choix de représentations graphiques produites à une période de l'histoire où la " conquête du passé ${ }^{2}$ " s'opère aussi bien par l'archéologie que par l'histoire de l'art, disciplines toutes deux en leur naissance. Par musées de papier, il faut entendre à la suite du savant, collectionneur et mécène Cassiano dal Pozzo, ces recueils « rassemblant, sous forme de gravures et de dessins, des représentations figurées d'œuvres antiques ${ }^{3}$ » formés par les collectionneurs

1 "Musées de papier : l'Antiquité en livres (1600-1800)", "Le Louvre au temps des Lumières ", "L'Antiquité rêvée : innovations et résistances au XVIII siècle ", "FrantzXavier Messerschmidt».

2 La formule est d'A. Schnapp : La Conquête du passé : aux origines de l'archéologie, Paris, Éditions Carré, 1993.

3 É. Décultot, Introduction du catalogue de l'exposition, Paris, Louvre éditions, 2010, p. 8. Nous suivons la nomenclature de ce catalogue : Fig. pour les illustrations des études liminaires, Cat. pour les œuvres exposées. 
des XVII et XVIII e siècles dans l'idée de constituer un "musée imaginaire ", enrichi d'une étude savante. C'est dire si l'objet, à la fois immatériel (en tant que discours) et concret (en tant que livre), ressortit pleinement à la représentation, en s'offrant comme le lieu de projections intellectuelles et un reflet des idées scientifiques et/ou esthétiques de ses concepteurs. À commencer par cette hésitation épistémologique entre ce qui, au début de l'Âge classique, ne se présente plus tout à fait comme un catalogue de collections (par sa spécialisation et son ordonnancement) et ce qui annonce le livre d'art (avec sa facture élégante et ses illustrations recherchées).

Ce qui frappe devant ces musées de papier, lesquels relèvent pour la plupart de la catégorie du livre imprimé, ce sont les compromis dont par-delà leur indécision générique ces ouvrages semblent la manifestation, compromis touchant à leur fonctionnement pratique et intellectuel : hésitation entre l'objet privé et l'objet "public ", affirmation conjointe d'un goût individualiste pour la propriété et d'une volonté militante de divulgation, tentation fréquente de commenter la pièce unique par le truchement d'un discours " universalisant ", etc. Au point qu'il paraitrait presque abusif de les considérer comme un ensemble homogène, tenant lieu au sens propre d'une réalité autre et définissant un type particulier d'ouvrage : un musée, de papier. Une caractéristique formelle semble pourtant rattacher ces livres à une même catégorie : le recours privilégié au format in-folio, comme si celui-ci pouvait cristalliser et neutraliser à la fois en son sein toutes les ambiguïtés. Certes l'histoire du livre artisanal nous apprend que la nature d'un texte conditionne souvent son support, les grands domaines de la pensée se distribuant peu ou prou selon les principaux formats de publication ${ }^{4}$; néanmoins, il semble que le recours au grand format s'enrichisse ici d'un supplément de signification en assumant une part, symbolique, du discours antiquaire, qu'il contribue à légitimer. Comme si la communauté d'esprit à l'initiative de ces ouvrages trouvait à s'exprimer aussi dans leur matérialité, marquée par des dimensions hors norme.

Envisageant les musées de papier du point de vue des matérialités du livre, ainsi qu'il est convenu d'aborder aujourd'hui l'objet-livre, nous voudrions observer la manière dont les scénographies calculées de l'in-folio, au cœur desquelles l'illustration trouve logiquement sa place, procèdent et offrent en même temps un reflet de la pensée antiquaire, et plus largement d'un nouveau rapport à la connaissance du passé. Ou pour le dire autrement, comment le tropisme de l'in-folio, format coutumier des livres rares, passant outre les contradictions internes de ces deux champs émergents du savoir sur l'Antiquité que sont l'archéologie et l'histoire de l'art, contribue à fonder l'autorité d'un discours propre, de nature scientifique.

4 Article "format " du Dictionnaire encyclopédique du livre, dir. P. Fouché et al., Paris, Cercle de la librairie, 2002-2011 ; voir aussi M.-H. BASsY, "Le texte et l'image ", in Histoire de l'édition française (II : Le livre triomphant [1660-1830]), dir. R. CHARTIER et H.-J. Martin, Paris, Fayard/Promodis, 1990, p. 182-183 - l'in-folio caractérise souvent le livre à figures. 


\section{Des livres d'images}

L'image est la justification première des musées de papier : elle en incarne le principe épistémologique par la remise en question de l'approche traditionnelle, philologique, du passé par les textes ${ }^{5}$ et la présentation figurée d'un matériau concret (objets, inscriptions, monuments, lieux), absent ou inaccessible (éloigné géographiquement, soustrait au regard, d'acquisition impossible, intransportable, disparu, etc.). En donnant à voir l'invisible, ainsi que l'a formulé K. Pomian dans ses travaux sur la culture de la curiosité ${ }^{6}$, elle ranime et fixe en même temps sa mémoire : elle le re-présente, le retrace en quelque sorte, en vertu d'une fonction d'abord " narrative " de l'illustration.

Aux côtés des pièces figurant dans les collections, l'image bidimensionnelle pourrait sembler appauvrir le rapport à l'objet en privant celui-ci de son volume. D'où, peut-être, ces rendus réalistes (jusqu'au trompe-l'œil) dont certaines reproductions explorent une large palette, appuyée sur les conventions du dessin académique : perspectives, jeux d'échelle et de proportions, modelé des formes, illusionnisme des ombres portées, variations chromatiques, parfois renforcés par les techniques de la camera obscura, comme si l'objet devait apparaître à la fois in pictura et realite, réifié par sa représentation ${ }^{7}$. Répondant à une intention archivistique, l'image revêt ici un caractère quasi "photographique ", servi par une gravure précise et de qualité. Or parce que l'antiquaire se veut un observateur rigoureux, l'image présente également les caractères d'un dessin «technique " à visée analytique. Fréquentes sont ainsi les illustrations qui ne visualisent pas seulement l'objet mais en montrent les détails, selon plusieurs points de vue, à l'instar des études anatomiques inspirées de la statuaire ${ }^{8}$ ou encore des relevés d'architecture qui modélisent un monument aux moyens conjoints de plans, projec-

5 Sauf quand les sources écrites sont lacunaires, ou absentes, comme dans l'archéologie de l'époque médiévale (voir Cat. 15, p. 86, la démarche de Gaignières pour son recueil d'Antiquités de la Gaule).

6 Collectionneurs, amateurs et curieux. Paris, Venise : XVI -XVIII siècle, Paris, Gallimard (Bibliothèque des Histoires), 1987, p. 15-59 ("Entre l'invisible et le visible : la collection»).

7 Dans la préface du tome III de son Recueil d'antiquités égyptiennes, étrusques, grecques et romaines (Paris, Desaint \& Saillant, 1759), Caylus présente la maîtrise du dessin comme un préalable à la juste saisie du " goût d'un siècle et d'une Nation » (p. vij), une grammaire au défaut de laquelle celui qui n'est pas "né au milieu des Antiquités " (p. xxiij) ne peut rivaliser avec le "tact " et l'" instinct " de ses homologues italiens, favorisés par leurs origines. Substitut (ou pis-aller) de l'observation directe, le dessin se justifie en outre scientifiquement, en autorisant les rapprochements visuels, fondement de l'archéologie comparative.

8 Voir les trois dessins du Torse du Belvédère pour le Museo Pio-Clementino d'E. Q. Visconti (catalogue, Fig. 5, p. 40). 
tions, coupes, vues cavalières, etc. ${ }^{9}$. La démarche est récurrente chez Caylus, dont le Recueil d'antiquités propose plusieurs planches structurées selon la même vue analytique, à savoir une représentation d'ensemble placée au centre de la page et entourée de projections variées (face, arrière, dessus, côté) et/ou de vues de détail ${ }^{10}$. L'image pour voir ne permet donc pas seulement de reconnaître, ou identifier : elle est aussi avancée comme un instrument de savoir, contribuant à la mise en évidence des sources authentiques, érigées en preuves historiques ${ }^{11}$. Partant, elle constitue un élément fondamental du dispositif critique.

Car l'image des musées de papier ne saurait oublier d'être image pour étudier, d'où son rapport étroit au texte, quand elle ne développe pas un discours autonome : au lecteur alors de déchiffrer et interpréter. Dans les musées de papier qui ne se limitent pas à une collection de figures (portefeuille, album ou recueil), un dialogue se noue entre l'image et le texte, qui donne son sens plein au concept d'illustration en tant que moyen d'accroître l'intelligibilité d'un discours. Lorsqu'elle prolonge le texte, l'image antiquaire explique, commente ${ }^{12}$ : c'est l'illustration "philologique " de Montfaucon, permettant de "v[oir] dans les images des histoires muettes que les anciens auteurs n'apprennent pas $^{13}{ }$; inversement, c'est le texte qui vient parfois compléter l'image, et développer ce qu'elle ne suffit pas à mettre en évidence. Texte et image s'éclairent ainsi réciproquement. Lorsqu' elle est argument au service du propos, l'illustration démontre, interprète selon des « conjectures vraisemblables ${ }^{14}$ ": c'est Perrault abondant le texte

9 Voir certaines planches d'architecture de J.-B. Séroux d'Agincourt dans son Histoire de l'Art par les monuments depuis sa décadence au IVe siècle jusqu'à son renouvellement au XVI $I^{e}$ siècle (Fig. 9, p. 32).

10 Voir le Fragment d'une très ancienne statue égyptienne (Cat. 21, p. 101) ou encore les trois étrusques de la planche XXXI du Recueil d’antiquités (Fig. 8, p. 31).

11 Ainsi le formule F. BIANCHINI dans son Istoria universale provata con monumenti e figurata con simboli degli antichi (Roma, A. de Rossi, 1697 ; cité par A. Schnapp dans La Conquête du passé, p. 186), en conclusion d'une démonstration de la supériorité des témoins archéologiques sur les peintures à sujet antique, de Raphaël ou du Titien par exemple : « [...] ces images-là font sur l'âme une impression bien plus profonde parce qu'elles n'attirent pas seulement les yeux par la séduction et la texture du dessin, mais parce qu'elles s'insinuent dans l'esprit avec ces signes évidents de l'antiquité qui servent de témoignage à la chose représentée. "

12 Son légendage éventuel peut d'ailleurs être vu comme une scorie de sa dépendance au texte, ou la prise en charge pédagogique d'une partie de celui-ci, comme dans le dispositif imaginé par $\mathrm{Cl}$. Perrault pour atténuer les inconvénients de lecture des " grandes figures " de ses Dix Livres d'architecture de Vitruve (Paris, Coignard, 1673) : " Pour [y] suppléer [...], on a mis auprès de chacune une explication, qui répète ce qui est à propos de cela dans le texte et dans les notes, dont le discours ne se pouvait pas rencontrer au droit des figures » (p. xvj [Préface]).

13 Dom B. De Montfaucon, L'Antiquité expliquée et représentée en figures, t. I, Paris, Delaulne et al., 1719, p. x (Préface).

14 CaYlus, Recueil d'antiquités, t. I, p. xiij (Avertissement). 
déficient ou corrompu des Dix Livres d'architecture de Vitruve par des figures glissées aux endroits les plus obscurs du texte, ce " afin de donner plus de facilité au lecteur de juger de la traduction, et lui laisser la liberté et le moyen d'en faire une autre si la nôtre ne lui agrée pas ${ }^{15}$ ». Agrément du texte enfin, l'image accompagne un discours savant réputé austère, offrant respiration ou écho ludique à l'exposé : ce sont les vignettes du Recueil d'antiquités de Caylus, qui préferent aux ornements typographiques de série des imprimeurs ce que l'on pourrait, sur le modèle des gravures tirées de tableaux, appeler des "bois d'interprétation ", s'appropriant artistiquement des antiques issues de collections connues (celle de Peiresc, par exemple) ${ }^{16}$.

Ce dernier exemple montre que l'image des musées de papier ne refuse pas toujours de s'adresser à la pensée esthétique, de ses spectateurs comme de ses auteurs. Pour Montfaucon, acquis au principe horatien de l'utile dulci, il importe que l'impressionnant appareil iconographique de l'Antiquité expliquée et représentée en figures soit " de belle grandeur, afin qu'on en puisse mieux remarquer toutes les parties, et qu'elles frappent davantage l'imagination ${ }^{17}$ "; et ne sont pas rares les illustrateurs qui, extrapolant les données dont ils disposent, laissent libre cours à des projections subjectives ou de pure fantaisie, démarche dont certaines vedute de Piranèse dans ses Antichita Romanae témoignent bien. Image pour voir et pour étudier, l'image est donc encore image pour rêver, voire sublimer, l'Antiquité, inscrivant ici les musées de papier dans le sillage des Wunderkammern du XVI ${ }^{\mathrm{e}}$ siècle, qui donnaient à voir un monde "poétisé ", recréé esthétiquement dans un rassemblement tout subjectif de pièces disparates ${ }^{18}$. Des " merveilles » humanistes, les musées de papier prolongent bien l'esprit, invitation à un voyage iconographique dans le temps et dans l'espace, qui préfigure en même temps nos modernes guides de voyage illustrés - pensons à ces relations de voyages d'antiquaires issus de la culture du Grand Tour ou de missions diplomatiques transformées en vastes enquêtes archéologiques, tels le Voyage d'Italie de Spon et Wheler, ou les Antiquités étrusques d'Hamilton publiées par d'Hancarville ${ }^{19}$.

On peut ainsi estimer que les riches illustrations des musées de papier participent à la définition d'un «style bibliographique antiquaire ", que résumerait la triade " voir,

15 Les Dix Livres d'architecture, p. [xvj] (Préface).

16 Recueil d'antiquités, t. I, p. xiv (Explication des vignettes et des culs-de-lampes).

17 L'Antiquité expliquée, p. x-xj (Préface). Fonction similaire à celle de l'hypotypose rhétorique, censée placer "sous les yeux " du spectateur une image vive et frappante, source d'intense émotion.

18 Au cœur de l'anticomanie du XVIII ${ }^{\mathrm{e}}$ siècle, ce principe d'un " collage " affranchi de toute préoccupation de vérité historique se retrouve chez des peintres comme Hubert Robert, ou encore chez les concepteurs imaginatifs de «fabriques de jardins ».

19 J. Spon, G. Wheler, Voyage d'Italie, de Dalmatie, de Grèce et du Levant, Lyon, Cellier le fils, 1678 (notons ici le choix du format in-douze, peu courant) ; P.-Fr.-H. D'HanCARVILLE, Antiquités étrusques, grecques et romaines, tirées du cabinet de M. Hamilton, envoyé extraordinaire et plénipotentiaire de S. M. Britannique en la Cour de Naples, Naples, [s. n.] [Morelli], 1766-1767 [1767-1776]. 
étudier, rêver ${ }^{20}$ ». Or illustrer, c'est également rendre illustre ${ }^{21}$, programme honoré dans le même temps par ces livres qui s'exhibent comme des publications de prestige, parfois d'apparat.

\section{Des livres de prestige}

Les musées de papier se présentent comme des ouvrages somptueux, signe tangible de leur rareté, hors chiffres de tirage observés, parfois élevés ${ }^{22}$. Cette apparence de luxe tient à leurs format, papier, reliure, illustrations et typographie, ainsi qu'à une mise en valeur du texte et de l'image reposant sur d'habiles stratégies de mise en page, et à une architecture d'ensemble attentive au cheminement intellectuel du lecteur. De fait, une véritable mise en scène de la pensée antiquaire est à l'œuvre dans ces ouvrages, qui en souligne la distinction pour mieux accompagner sa promotion.

Le musée de papier est un livre onéreux, au regard déjà de son format exceptionnel et du nombre de volumes, sources de dépense importante en papier et reliure. Rien d'étonnant dans ces conditions à le voir recourir parfois au système de la souscription tant il parait opportun d'" aid[er] aux frais immenses qu'une telle entreprise entraîne avec elle ${ }^{23}$ ", voire opter pour un financement à compte d'auteur, comme le fait Winckelmann pour ses Monumenti antichi ${ }^{24}$. Ce grand livre est aussi un beau livre qui parle de sa destination privilégiée : le cabinet ou la bibliothèque, à savoir un espace choisi inspirant la déférence et impliquant un certain mode de lecture ou de consultation, marqué par la solennité sinon un cérémonial. L'on imagine assez le rythme de lecture imposé par de tels ouvrages, l'in-folio ouvert tendant à l'in-plano, de maniement peu aisé, et son feuilletage se trouvant compliqué par la lecture conjointe du texte et de l'image : Perrault le déplore, Montfaucon s'en félicite ${ }^{25}$. Par leur richesse quantitative

20 Formulation empruntée au titre de la contribution de J.-R. Gaborit au catalogue de l'exposition D'après l'antique organisée par le Louvre en 2000-2001 (dir. J.-P. Cuzin, J.-R. Gaborit et A. PASQUIER, Éditions RMn, 2000).

21 Dictionnaire de l'Académie françoise, Paris, Vve Coignard et Coignard, 1694.

22 C'est le cas notamment de l'Antiquité expliquée de Montfaucon, avec un premier tirage à 1800 exemplaires et une réédition quasi immédiate à 2200 (cf. C. Grell, Le Dix-huitième siècle et l'Antiquité en France, 1680-1789, Oxford, Voltaire Foundation, 1995, t. I, p. 247-248).

23 P.-Fr.-H. D'Hancarville, Antiquités étrusques, p. [i] (Avertissement). Sur la souscription comme outil de maitrise des coûts de publication, cf. W. KIRSOP, "Pour une histoire bibliographique de la souscription en France au XVIII siècle ", in Trasmissione dei testi a stampa nel periodo moderno, Roma-Viterbo 27-29 giugno 1985, Roma, Edizioni dell'Ateneo, 1987 , p. $255-282$.

24 Monumenti antichi inediti, spiegati ed illustrati, Roma, a spese dell'autore, 1767.

25 Respectivement dans Les Dix Livres d'architecture, p. xvj (Préface) et L'Antiquité expliquée, p. vj (Préface). 
et qualitative, les illustrations tendent elles-mêmes à suspendre le mouvement continu de la lecture en captant le regard, au risque d'en faire oublier ce qui fonde ce type d'ouvrage : l'association de l'image à une dissertation ${ }^{26}$. Richesse en nombre : hors figures insérées dans le texte et proportionnellement à l'étendue de chaque projet, ces ouvrages se signalent toujours par la quantité de gravures, remarquable au sein même du genre luxueux qu'est le livre illustré. Richesse par le caractère unique des dessins originaux de certains recueils 27 , ou par la haute facture des gravures, qui recourent à quelques grands noms de l'estampe (Cortone, Buonamici, Mellan, Bouchardon, Bosse, Audran, Cochin, Le Bas, etc. ${ }^{28}$ ) et prévoient parfois des finitions à l'exemplaire, comme ces planches du Recueil des peintures antiques trouvées à Rome imitées de dessins de Bartoli que Caylus et Mariette firent peindre à gouache, sous leur contrôle, par chaque destinataire des trente exemplaires de l'édition, à ce titre déjà exceptionnelle ${ }^{29}$. Quant aux stratégies typographiques en jeu, elles impliquent à l'évidence un travail spécifique de l'atelier d'imprimerie, logiquement répercuté sur le prix final : composition sur plusieurs colonnes, encadrée, bilingue, assortie de notes infra-paginales et/ou marginales, de tableaux synoptiques, insertions de transcriptions épigraphiques, recours à des vignettes spéciales (tirées de bois ou gravures uniques), etc. Curieux paradoxe pour ces imprimés qui multiplient les signes d'une singularité jusqu’à imaginer une appropriation personnalisée, mais se disant toujours soucieux de livrer à un large public les secrets jalousement gardés des cabinets...

Le prestige attaché aux musées de papier se lit encore dans les mises en page de l'illustration, du texte, et le cas échéant dans leur mise en relation sur une même page. Pièce maîtresse du dispositif, la planche iconographique, déjà différenciée par son impression à part, tend à se distinguer encore par son déploiement obligé lorsque l'objet figuré déborde le cadre de la page (plans de villes, mosaïques, fresques, frises). En général, il suffit d'ouvrir le bifolium caractéristique de l'in-folio mais des montages sophistiqués sont parfois rendus nécessaires - tels les feuillets accolés de la vue

26 Dans ses Antiquités étrusques (p. [i], Avertissement), D’Hancarville glisse non sans ironie que les illustrations de l'ouvrage seront " aussi propres à meubler un appartement qu'à enrichir un portefeuille d'estampes ou à tenir leur place dans une bibliothèque ».

27 Voir les dessins de P. S. Bartoli reliés avec des textes manuscrits du XVIII ${ }^{\mathrm{e}}$ siècle par Caylus et Mariette (Cat. 6, p. 58).

28 Respectivement pour les illustrations du Museo cartaceo de Dal Pozzo (Cat. 1, p. 50), de la Camera ed inscrizioni sepulcrali de Bianchini (Cat. 9, p. 70), des Tableaux du Cabinet du Roy de Félibien (Cat. 22, p. 102), du Traité des pierres gravées de Mariette (Cat. 27, p. 112), des Mémoires pour servir à l'Histoire naturelle de Perrault (Cat. 30, p. 118), des Proportions du corps humain d'Audran (Cat. 32, p. 122), des Observations sur les antiquités de la ville d'Herculanum de Cochin et Bellicard (Cat. 37, p. 133), des Ruines des plus beaux monuments de la Grèce de Le Roy (Cat. 39, p. 136).

29 Recueil des peintures antiques trouvées à Rome, imitées fidèlement pour les couleurs et le trait d'après les dessins coloriés par Pietro Santi Bartoli, Paris, [s. n.], 1757. 
panoramique de Palmyre dans l'ouvrage de Wood ${ }^{30}$ - ou conçus pour accompagner l'imagination : le lecteur du Palazzo de Cesari de Bianchini, dépliant les volets du vaste plan reconstitué d'un palais impérial du Palatin, crut-il un moment embrasser, porté par le point de vue "à vol d'oiseau " du dessin de Nicoletti, les fastes de l'époque impériale ${ }^{31}$ ? L'on ne peut s'empêcher ici de faire le lien avec nos modernes " pop-up ", dont les pliages complexes, retenus dans l'espace plan du livre fermé, font soudain en s'ouvrant surgir sous les yeux la «3D ». Quel que soit son objet, la planche obéit par ailleurs à une structure visuelle forte, dont la syntaxe emprunte à une géométrie classique de l'image : partition de l'espace selon les lignes médianes, symétries, oppositions, champ/contre-champ, premier plan/arrière-plan, etc., comme s'il importait de marquer la présence d'un ordre de la connaissance, jusque dans les semis (faussement) aléatoires d'objets ou les juxtapositions elles aussi pensées de figures ${ }^{32}$. Organisée, l'image antiquaire s'adresse donc d'abord à qui sait en décoder les règles visuelles, parce que familier de tels codes, à travers le modèle de la grande peinture notamment. La présence fréquente d'un ou plusieurs filets typographiques, éventuellement renforcés d'une large marge, rappelle d'ailleurs a priori ses affinités avec l'art pictural, ici conçu comme art de prestige $^{33}$. Il n'est pas jusqu'au texte qui ne ménage lui aussi des effets typographiques (emploi des caractères de civilité, par exemple) pour se singulariser, et désigner le musée de papier comme un objet extra-ordinaire dans la classe des livres illustrés ${ }^{34}$. Dans le rapport même du texte et de l'image, parfois très " graphique ", s'affirme encore cette recherche esthétique, comme on a pu en juger à travers la mise en regard d'un croquis de Jacob Spon et de sa version imprimée pour les Miscellanea eruditae antiquitatis ${ }^{35}$.

Malgré leur présence attendue dans un livre "savant ", ces seuils hautement signifiants que sont le frontispice et l'avant-propos viennent eux aussi réaffirmer le caractère exceptionnel des musées de papier, en amplifiant chacun selon ses codes leur autopromotion : la culture de l'emblème pour le frontispice, la rhétorique du contrat de lecture pour l'avis au lecteur ou la préface. Pièce majeure du dispositif iconographique, le fron-

30 R. Wood, The Ruins of Palmyra, otherwise Tedmor in the Desart, Londres, Millard, 1753 (Cat. 36, p. 131).

31 F. Bianchini, Del Palazzo de' Cesari, Vérone, Berno, 1738 (Cat. 10, p. 72).

32 Mentionnons en exemple les séries de figures caractéristiques des planches de Séroux d'Agincourt pour son Histoire de l'art par les monuments, justifiées par une démarche comparatiste (Cat. 47, p. 152).

33 Une telle présence d'influences picturales est sensible dans les planches des Gemmae antiquae caelatae de Ph. von Stosch (Amsterdam, Picard, 1724 ; Cat. 26, p. 110), dont les arrangements visuels reprennent certains codes du portrait gravé, en même temps que du relief monumental sculpté.

34 Cf. le Voyage pittoresque ou Description des Royaumes de Naples et de Sicile de l'abbé de Saint-Non (Paris, [Clousier], 1781-1786), avec sa dédicace gravée en "lettres façon d'écriture » et un style typographique s'inspirant ostensiblement de celui de l'Imprimerie Royale.

35 Lyon, Amaulry, 1685 (Cat. 35, p. 128). 
tispice concrétise l'idée de majesté à travers un discours allégorique certes convenu mais dont les éléments sont d'autant plus parlants ici qu'ils recourent volontiers à des architectures monumentales rappelant les vestiges de l'Antiquité : les exemples ne manquent pas, de la Roma Sotterranea de Bosio (arc de triomphe revisité par le style renaissant) au Recueil d'antiquités de Caylus ("bagatelle » de jardin à colonnes de style dorique) en passant par L'Antiquité expliquée de Montfaucon (paysage de fabrique mêlant galerie à arcades, portique de style corinthien et éléments architectoniques "à l'antique ") ${ }^{36}$. Visant un lecteur rompu aux codes de l'image allégorique, ces frontispices s'exposent avec un supplément de sens, renvoyant à la démarche intellectuelle même des antiquaires, qu'ils glorifient, ainsi qu'à son objet, doté d'une dignité intrinsèque. De même la plupart des propos liminaires développent-ils un discours de l'exceptionnel, outrepassant l'habituelle rhétorique de propagande publicitaire pour esquisser une problématique scientifique, expliquée et vérifiée ensuite par le corps de l'ouvrage : la frontière semble alors ténue entre le mode d'emploi d'un manuel d'antiquités (éventuellement complété d'un appareil d'indices et de tables) et l'esquisse théorique dévoilant les grands contours d'un projet d'ensemble. Frontispice et avant-propos sont ainsi à proprement parler deux entrées en matière, deux seuils symboliques informant le lecteur de la nature " inédite » du lieu qu'il s'apprête à pénétrer - le musée de papier n'est ni tout à fait un musée, ni tout à fait un livre d'images -, tout en lui conférant préalablement le statut de lecteur choisi, privilégié (i. e. nanti d'un privilège, bien sûr exorbitant).

"Livres-spectacles ", les musées de papier installent puis confortent au fil de leurs pages un climat de grandeur, sursignifié par la majesté de l'in-folio, sans jamais cependant que cette somptuosité ne tombe dans le luxe factice. Parce qu'ils veillent à leur propre éloge, sur le mode rhétorique de l'amplification et au moyen d'un système iconique et textuel pensé pour donner à voir et faire éprouver leur distinction, ils ressortissent au fond au genre épidictique ; et par ce truchement, la démarche antiquaire entend bien faire valoir ses lettres (dûment imprimées) de noblesse, qui la démarquent des autres livres d'antiques que sont les simples catalogues de collections. L'ambition ne fait guère de doute : il s'agit, à la mesure du format imposant (qui s'impose et en impose) de l'in-folio, de faire procéder la référence de la révérence.

\section{Des livres de référence}

La valeur scientifique des musées de papier découle de leur irréductibilité à la catégorie $\mathrm{du}$ « beau livre » proposant une approche de l'Antiquité fondée sur la seule émotion

36 Particulièrement élaborée reste l'illustration de la Demonstratio Historiae Ecclesiasticae de Bianchini (Cat. 11, p. 76), pièce maîtresse de l'exposition du Louvre : composition d'ensemble et éléments constitutifs y cristallisent toutes les projections intellectuelles que pouvaient accueillir les musées de papier, entre représentation d'un musée réel et réunion d'une collection virtuelle dans un livre d'apparat. 
esthétique. Pour comprendre l'admiration suscitée par ces ouvrages, il faut s'interroger sur leurs usages et leur public, voire sur l'imaginaire développé autour d'eux : de quelle reconnaissance les auteurs bénéficient-ils à travers leur lecture, et quelle influence leur discours exerce-t-il dans le champ de l'antiquarisme?

Les musées de papier figurent en priorité chez les antiquaires, ces "savants dans la connaissance des antiques et qui en [sont] curieux " tels que les définit la première édition (1694) du Dictionnaire de l'Académie française. Catégorie en fait générique (qui réunit les " amateurs ", " connaisseurs » et autres " érudits "), où se côtoient deux visages de l'antiquaire appelés à se différencier puis à s'opposer au fur et à mesure qu'évolue la science du passé : le collectionneur, que motiverait la seule jouissance de l'objet, et le savant, animé par une véritable démarche scientifique, historienne. Le mot " curieux " renvoie évidemment aux cabinets de curiosités de la Renaissance. Il laisse imaginer l'intimité de ce lieu où l'on "serr[e] des papiers, des livres, ou quelque autre chose selon la profession ou l'humeur de la personne qui y habite ${ }^{37}$ " et dans lequel l'amateur de loisir érudit se retire pour le plaisir solipsiste de " voir, apprendre, posséder ${ }^{38}$ ". Pour ce collectionneur-là, " homme par excellence de [...] la concupiscence visuelle ${ }^{39}$ ", le musée de papier, avec son apparat exceptionnel d'illustrations, représente une façon d'augmenter virtuellement ses trésors en satisfaisant à coût limité sa passion, immodérée par nature, de la possession privée ${ }^{40}$ - et en ce sens, le musée de papier constitue un intéressant témoin de l'histoire des cabinets bibliophiliques de livres rares en rapport avec l'évolution du goût collectionniste. Cependant, compte tenu des mécanismes profonds de tout secret, il est aussi inscrit dans le fonctionnement du mundus conclusus de l'antiquaire de cabinet de s'ouvrir au commerce des autres hommes, en suscitant au minimum la citation, plus largement le commentaire. Livre de prestige, voué à une mise en valeur, le musée de papier joue ici un rôle culturel comme vecteur important de la sociabilité antiquaire - il en est aussi un produit en ce qu'il résulte de sollicitations et contributions diverses (de " fouilleurs ", voyageurs, correspondants, collectionneurs, artistes, imprimeurs, marchands d'art, libraires, etc.). Destiné au pupitre (selon l'usage des grands ouvrages de référence proposés à la consultation), peu transportable car encombrant, le musée de papier s'expose non sans ostentation en une théâtralisation préméditée de sa rareté, scruté comme telle autre pièce de collection, touché, commenté, raconté, convoité, copié, imité, emprunté... Bref, ce livre précieux invitant à sa thésaurisation connaît aussi un destin " public ", bien que cette vocation reste circonscrite à un cercle de happy few liés par une communauté d'intérêts intellectuels.

37 Académie, 1694 (art. « Cabinet»).

38 Ibid. (art. "Curiosité »).

39 J. CLAIR, "La mélancolie du savoir ", in Mélancolie, génie et folie en Occident, catalogue de l'exposition du Grand Palais (Paris, Gallimard, 2005), p. 202.

40 Tant il est vrai que « la curiosité n'est pas un goût pour ce qui est bon ou ce qui est beau, mais pour ce qui est rare, unique, pour ce qu'on a et ce que les autres n'ont point " (LA BRuYÈre, Caractères, " De la mode ", 2). 
Or en s'appropriant ainsi le musée de papier, ce réseau de connaisseurs s'approprie aussi collectivement la pensée qui le sous-tend et affirme sans relâche son caractère inédit.

Il est frappant, laissant de côté la rhétorique ordinaire des discours préfaciels, de voir à quel point les auteurs des musées de papier se montrent attachés à se camper en pionniers d'une approche intellectuelle originale, et comment le prestige de l'infolio vient conforter pour eux ce caractère de nouveauté. Sans faire entièrement table rase de la tradition philologique - dans un premier temps, la convocation des vestiges historiques n'est qu'une simple mise à l'épreuve des textes ${ }^{41}$-, cette nouvelle approche s'en démarque pourtant sur un double plan, épistémologique et méthodologique, puisqu'il s'agit d'asseoir la connaissance (historique et esthétique) de l'Antiquité sur une critique rationnelle des sources non textuelles, au moyen d'instruments et de techniques inconnus de la glose littéraire. Et l'auteur-antiquaire de légitimer sa position par l'exposé d'un système descriptif qu'on pourrait dire "positif ", avec ses typologies et nomenclatures, tout en promouvant la prospection géographique, l'épigraphie, la numismatique, la topographie, etc. comme autant d'outils pertinents d'interprétation scientifique du passé. Pour le coup, le musée de papier peut bien s'octroyer le mérite d'être un livre d'antiques d'un nouveau genre puisque l'image, motivée intellectuellement par cette démarche "technique ", y acquiert un même poids scientifique que dans les traités de médecine, astronomie, etc. ; dans le format choisi de l'in-folio d'autre part, l'on peut voir comme un brevet supplémentaire de scientificité décerné à un propos encore mal situé dans le champ épistémologique. De là sans doute cette tendance des auteurs de musées de papier à se poser en figures d'utilité publique, s'adressant volontiers aux non-initiés ; posture altière s'il en est, justifiant que le " connaisseur " (et non plus le "savant ") puisse assumer parfois une fonction d'expertise - et l'on sent bien ce sentiment de supériorité chez un Caylus "mécène du roi ${ }^{42}$ ", par exemple. L'écart paraît ici consommé avec la figure du collectionneur humaniste, dont le penchant pour l'ascèse et la dilection narcissique relèvent d'un tout autre ordre, celui de la vanité, en fait disqualifiée par la pensée antiquaire : "La vanité n'étant point mon objet, [...] je ne fais point un cabinet, je fais un cours d'Antiquité et je cherche les usages, ce qui les prouve, les pratiques, ce qui les démontre ${ }^{43}$ ", dit encore Caylus. Dès lors se dévoile l'enjeu profond du musée de papier : consacrer l'autorité d'un " autre " discours sur l'histoire et à travers lui, le pouvoir d'une nouvelle classe d'intellectuels sûre de sa légitimité dans la maîtrise du passé à partir du moment où celle-ci peut faire la preuve de sa valeur scientifique. Notons que cette autorité peut jouer à des niveaux

41 CaYLus, Recueil d'antiquités (Préface) : "Lorsque j'ai commencé à faire graver cette suite, j'ai eu d'abord en vue l'homme de lettres qui ne cherche dans les monuments que les rapports qu'ils ont aux témoignages des Anciens. "

42 Caylus, mécène du roi. Collectionner les antiquités au XVIII siècle, catalogue d'exposition de la BnF, dir. I. AGHION, INHA, 2002.

43 Lettre du 12 janvier 1758 à l'antiquaire italien Paciaudi, citée par les Goncourt dans leurs Portraits intimes du XVIII siècle (Dentu, 1858, p. 26). 
différents de pouvoir, l'influence de l'antiquaire de cour ne s'exerçant certes pas selon les mêmes enjeux et modalités que celle de l'érudit impliqué dans une communauté plus restreinte, traversée par d'autres déterminations ${ }^{44}$; mais il est remarquable que le format de l' in-folio s'impose en toutes circonstances comme le support naturel, concrètement et symboliquement, de l'autorité du discours antiquaire saisi dans sa démarche d'appropriation du passé. Quelle que soit l'origine de ce discours, princière ou non, française ou italienne.

Ainsi, ce qu'offre la monumentalité de l'in-folio à ce discours "inédit " mais professé avec assurance, c'est un espace d'accaparement d'un pouvoir, assez vaste pour accueillir la libre expression d'une pensée «nouvelle » d'emblée sacralisée. Pour autant, ce discours est-il si différent de celui des glosateurs des sources écrites? Dans les scrupules des antiquaires du XVII ${ }^{\mathrm{e}}$ siècle - et cela vaut pour le XVIII ${ }^{\mathrm{e}}$-, A. Schnapp l'a bien montré, une "pensée du texte " demeure à l'œuvre, et l'« on distingue clairement la métaphore philologique qui tend à constituer les systèmes d'objets en systèmes de langues ${ }^{45}$ ». Moyennant quoi, l'in-folio du musée de papier éclairerait d'abord la connivence pérenne de l'antiquaire avec le livre, d'autant plus symbolique que celui-ci se veut rare et recherché ${ }^{46}$.

Rendant compte du phénomène du collectionnisme, qu'il envisage sous l'angle privilégié de l'histoire culturelle, K. Pomian a élaboré une théorie des sémiophores, ou " objets porteurs de significations ", directement applicable aux musées de papier. Situés à la croisée d'orientations diverses, traditionnelles ou innovantes, de la démarche antiquaire à l'Âge classique (philologie, archéologie, histoire de l'art), creuset dynamique de projections intellectuelles stimulées par l'ambiguïté de leur statut générique et l'indécision de leur destination, entre le cabinet d'antiques et la bibliothèque lettrée, ces musées, pièces de collection eux-mêmes, peuvent être regardés comme des sémiophores particulièrement riches de sens. Mais s'ils le sont, c'est aussi en tant que livres, tout livre se définissant déjà comme un système complexe de signes (sociaux, économiques, idéologiques, etc.), enrichi d'un second système dans le cas de l'in-folio. Fort de sa double identité, le musée de papier s'inscrit clairement dans deux économies : une économie réelle - c'est un livre de prix -, et une économie symbolique - c'est un livre rare. Livre précieux, d'un raffinement esthétique plus somptuaire parfois qu'immédiatement

44 Sur les accointances du collectionnisme princier avec les scenarii de pouvoir, voir la stimulante analyse de P. Falguières sur la manière dont la chambre des merveilles, lieu symbolique du corps inaccessible du prince, se définit par une sanctuarisation rigoureuse des signes de la domination (La Chambre des merveilles, Paris, Bayard (Le rayon des curiosités), 2003).

45 La Conquête du passé, p. 181.

46 Emblématique est en ce sens la présentation physique de la dactyliothèque de Ph.-D. Lippert (Fig. 8, p. 43 et Cat. 28, p. 113), dont les coffrets sont réunis dans des boîtiers imitant un grand in-folio luxueusement relié. 
utilitaire, il intéresse par la présence d'un important apparat d'illustrations, qui fournit en même temps sa justification intellectuelle; livre rare, autrement dit " qui n'est pas commun, ordinaire ${ }^{47}$ ", il fonde sa valeur sur l'idée de prestige, soulignée par une "scénographie " maîtrisée des désirs de lecture et d'acquisition. De ce fonctionnement particulier du musée de papier, le format de l'in-folio est une manifestation éclatante, jusqu'à l'hypertrophie du "grand in-folio " et/ou de l'édition en plusieurs volumes : il est constant en effet, même si cette rhétorique n'est pas exempte de fausse modestie, que le projet excédant les intentions premières des auteurs, ceux-ci s'avouent dépassés par la monumentalité de l'entreprise.

Or ni le prix ni la rareté ne sauraient être considérés comme des propriétés objectives du musée de papier puisque ces qualités procèdent d'un geste d'assignation de la valeur, centrée sur trois enjeux : conférer une distinction, marquer l'autorité d'un discours scientifique inédit, promouvoir une nouvelle élite intellectuelle. Objet sacralisé, le musée de papier élève à un rang éminent son possesseur et son lecteur, à la réputation desquels il contribue : il déchiffre donc socialement son public, s'érigeant en signe identitaire d'un groupe qui se reconnaît dans la pratique distinguée du musée et du livre, avec mise à l'écart des profanes ${ }^{48}$. Objet savant mis au service de la science antiquaire et de l'histoire de l'art, il se propose, dans une période d'érosion de l'érudition livresque, de substituer à la tradition philologique une critique des sources directes jugées plus authentiques; ce faisant, il entend bien fonder une nouvelle " autorité qui tienne lieu de raison positive ${ }^{49}$ ". Objet de référence enfin, le musée de papier révoque la culture humaniste de la curiosité érudite, qui " enterre ${ }^{50}$ " les collections dans les cabinets, pour lui préférer les formes ouvertes d'une culture mondaine convaincue des vertus citoyennes de la divulgation ; partant, il permet à une catégorie restreinte de " connaisseurs ", qui s'approprie l'Antiquité et la constitue en patrimoine commun placé sous son arbitrage, d'exhiber le musée de papier comme son manifeste intellectuel, un objet de pouvoir donc, pour ne pas dire un paradigme. Il serait en ce sens intéressant d'examiner les vicissitudes de l'affirmation de ce pouvoir, lorsque avec l'évolution des formats éditoriaux vers plus de maniabilité, l'in-folio imposant apparaît de plus en plus incongru, voire anachronique ; ou encore, comment réintégrant dans le champ de la connaissance de l'Antiquité le public tenu à distance par l'in-folio de prestige, les versions économiques des musées de papier (copies et contrefaçons), qui doublent parfois leurs modèles remarquables, réévaluent la notion même d'autorité du discours antiquaire par un autre regard porté sur l'idée de patrimoine commun. Au demeurant, l'ambiguïté la plus fascinante du musée de papier ne tient-elle pas,

47 Académie, 1694 (art. "Rare»).

48 Ibid. (art. "Profane " : "se dit fig[urément] d'une personne qu'on ne veut point admettre dans une societé ").

49 Perrault, Les Dix Livres d'architecture, p. 9 (Préface).

50 D'Hancarville, Antiquités étrusques, p. xix (Préface). 
justement, à son fonctionnement éditorial paradoxal : parler du livre avant que de son contenu, et en faire l'éloge à partir du postulat théorique d'un déchiffrement possible du passé sans les textes?

Magali Soulatges

24, rue Pradier

F-30 000 Nîmes

Université d'Avignon et des Pays de Vaucluse

Institut de Recherche sur la Renaissance,

l'âge classique et les Lumières (UMR 5186)

université de Montpellier 3

magali.soulatges@univ-avignon.fr 